\title{
The Peristaltic Motion inside a Vertical Cylindrical Tube Surrounded Vapour Bubble with Two-Phase Density Flow
}

\author{
S. A. Mohammadein ${ }^{1}$, A. K. Abu-Nab ${ }^{2}$, G. A. Shalaby ${ }^{2}$ \\ ${ }^{1}$ Department of Mathematics, Faculty of Science, Tanta University, Tanta, Egypt \\ ${ }^{2}$ Department of Mathematics, Faculty of Science, Menoufia University, Shebin El-Koom, Egypt
}

Email address:

ahmed.abunab@yahoo.com (A. K. Abu-Nab), selimali40_43@yahoo.com (S. A. Mohammadein), gamilshalaby550@yahoo.com (G. A. Shalaby)

\section{To cite this article:}

S. A. Mohammadein, A. K. Abu-Nab, G. A. Shalaby. The Peristaltic Motion inside a Vertical Cylindrical Tube Surrounded Vapour Bubble with Two-Phase Density Flow. Advances in Bioscience and Bioengineering. Vol. 5, No. 4, 2017, pp. 71-77. doi: 10.11648/j.abb.20170504.14

Received: March 21, 2017; Accepted: April 19, 2017; Published: October 19, 2017

\begin{abstract}
The paper presents the growth of vapour bubble in a viscous, superheated liquid. The growth of vapour bubble between two-phase density flow in a vertical cylindrical tube under the effect of peristaltic motion of long wavelength and low Reynolds number is studied. The mathematical model is formulated by mass, momentum, and heat equations. The analytical solution is obtained for temperature and velocity distribution under the effect of different physical parameters. The growth process is studied under the affected of density ratio $\varepsilon$ and amplitude ratio $e$. Moreover, the relation between the bubble radius $R$ with the density ratio $E$, and amplitude ratio $e$ are obtained. Theseresults agreement with some previous theoretical efforts.
\end{abstract}

Keywords: Peristaltic Flow, Heat Transfer, Grashof Number, Superheated Liquid, Growth of Vapour Bubble

\section{Introduction}

The transportation of bio-fluid by continuous wave like muscle contraction and relaxation of the wall of physiological vessels such as esophagus, stomach, intestines, sometimes in ureters, blood vessels and other hollow tubes is known as peristaltic transport $[1,2]$. Peristaltic force is an organic siphon which employs episodie wave-like squeezing movement which voyages down a vessel and forces the stuffing of the vessel. This mechanism also occurs in many applications involving bio-mechanical systems such as finger and roller pumps. Heart lung machines blood pump machines, dialysis machine and also transport of noxious fluid in nuclear industries [3]. In recent years, considerable efforts have been usefully devoted to the study of peristaltic flow of non-Newtonian fluids because a practical and fundamental constitutive relation that can be used for all fluids and flow is not available. Hayat et al. [4] investigated the effects of different wave forms on the peristaltic flow of micropolar fluids through a channel, and reported that the maximum pressure against which peristalsis works increases with the coupling number but decreases with the micropolar parameter. On the other hand, Single bubble growth on a solid surface was studied by generating a single bubble on a submerged heater. As heat is applied the liquid becomes supersaturated locally. This procedure is somewhat different to the traditional way to study these phenomena by degassing supersaturated liquid through decompression, where a single bubble alone cannot be produced. Thermal degassing which involves mass transfer but also heat transfer is thus achieved (as opposed to decompression degassing which only involves mass transfer in theory). To study the bubble generation and growth separately from the gravitational effects on them, the experiments where performed in microgravity conditions [5, 6]. The experimental observations were compared to a theoretical model [7] derived considering spherical bubbles in a uniformly cooled liquid that were heated from the inside. The initial stage of growth was shown to agree with a parabolic diffusion law, after which a linear growth model was more appropriate. The lateral motion of the bubbles along the heater as they are first generated was also looked at and discussed with respect to the surface of the heaters used [8]. Multiple bubble growth and detachment showed competition for the dissolved gas available in the supersaturated solution amongst bubbles growing closely together [9]. The final size of bubbles was shown to be smaller than that of a single bubble, and a critical 
temperature could be found above which any increase in temperature did not result in faster bubble growth. Karapantsios et al. have argued for the necessity to study the characteristics of bubbly flow (multiple bubbles flowing with the liquid) in addition to single bubble generation, since it is this abundance of bubbles which is at the origin of DCS above some threshold. An impedance spectroscopy technique, In Vitro Embolic Detector (IVED), was developed to detect bubbles in the blood stream by measuring the gas fraction. The in vitro phase of this project showed very good resolution as well as sensitivity to variations in gas fraction and bubble size in bubbly flows [10], and the in vivo phase, currently animal testing, is in progress. The results were validated through acoustic spectroscopy and electrical impedance tomography measurements. An in vitro experiment to simulate a realistic bubbly flow in the human vena cava was devised to investigate the effect of surfactant and electrolyte concentrations on the bubble size distribution (measured both by the IVED and electrical impedance tomography for validation purposes) to continue the improvement of these techniques but sized optically in this study [11]. The study found no correlation between the bubble size and the radial position of the tube or viscosity of the liquid. The size distribution was however found dependent on the flow rate and lower for higher surfactant and electrolyte concentrations and when both were added together this effect was amplified. An assumption throughout the paper is that the addition of surfactants will not affect the radial distribution of different bubble sizes in the tube, and all measurements for sizing were done near the surface of the tube. Another limitation of the study is the high bubble count needed for sizing. A mathematical study to look at the interaction between blood born bubbles and tissue bubbles was conducted, assuming that bubbles can form in tissues and in the wall of vessels $[12,21]$. Once again competition for dissolved gas was pointed out. It was also shown that the number of tissue bubbles will influence the number of blood bubbles, whereas the opposite effect is very unlikely. The main variable of interest is obviously perfusion of tissues. The phenomenon of competition for dissolved gas among growing bubbles was further investigated through numerical simulations [13]. A clamping phenomenon was demonstrated above a certain density of bubbles per unit tissue, after which the washout rate was considerably diminished, going from exponential to linear. This finding seems realistic since a number of decompression algorithms, the so-called exponential linear kinetics models, use a linear washout rate with. The theory of the growth of a single vapour bubble in a superheated liquid has been considered by several authors. The inertia controlled growth was presented by Rayleigh [14], who determined the first equation of motion for a spherical bubble growth (or collapse). The asymptotic solution, presented by Plesset and Zwick [15], considered thermal diffusion controlled growth, neglecting liquid inertia, and provided a zero-order approximate solution for the bubble wall temperature with the assumption of a thin thermal boundary layer with error of less than 10\% [3]. Their solution [16] was in good agreement with the experimental data of Degarabedian [18] in moderately superheated water up to $6^{\circ} \mathrm{C}$. Forster and Zuber [17] were basically in agreement with Plesset and Zwick [15]. A new treatment of the problem was presented by Scriven [19], who solved the energy equation without assuming a thin thermal boundary layer, and his asymptotic solution for moderate superheated liquid was identical to that of Plesset and Zwick [15]. Mikic et al. [20] effectively combined inertial and thermal diffusion controlled growth using the Clausius-Clapeyron equation. In this study, under the effect of heat transfer growth, the growth of a vapour bubble in a viscous, superheated liquid in a vertical cylindrical tube is studied. The analytical solution is obtained for temperature and velocity distribution under the effect of Grashof number $G_{r}$, heat source parameter $\beta$, and density ratio $\varepsilon$. The analytical solution of the heat and momentum equations are used to obtain a relation between bubble radius $R$ and time $t$, which takes into account the effect of some physical parameter.

\section{Analysis}

Consider the axisymmetric of a Newtonian fluid in circular cylindrical tube with a sinusoidal wave of small amplitude traveling down its wall is shown as in Fig. 1. The wall of the tube is given by the equation

$$
H(z, t)=a+b \sin \frac{2 \pi}{\lambda}(z-c t)
$$

where $a$ is the average radius of the original undisturbed tube, $b$ is the amplitude of the wave, $\lambda$ is the wavelength and $c$ is the wave speed. $r$ and $z$ are the cylindrical polar coordinates with $z$ measured a long the axis of the tube and $r$ is in the radial direction. Let $u$ and $w$ be the velocity components in the radial and axial directions, respectively.The peristaltic bubbly flow of a viscous incompressible Newtonian fluid through a vertical tube.

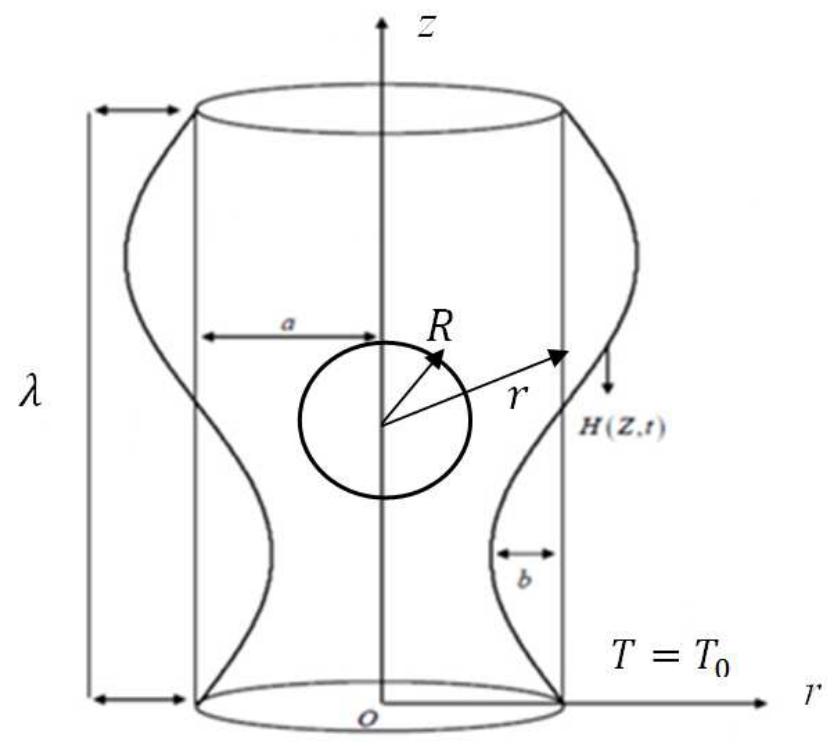

Figure 1. The Problem Sketch. 
The mathematical model of the physical problem is described by the conservation equations of mass, momentum, and heat transfer as follows:

$$
\begin{gathered}
\frac{1}{r} \frac{\partial}{\partial r}(r u)+\frac{\partial w}{\partial z}=0 \\
\rho\left(u \frac{\partial u}{\partial r}+w \frac{\partial u}{\partial z}\right)=-\frac{\partial P}{\partial r}+\mu\left\{\frac{1}{r} \frac{\partial}{\partial r}\left(r \frac{\partial u}{\partial r}\right)-\frac{1}{r^{2}}+\frac{\partial^{2}}{\partial z^{2}}\right\} u \\
\rho\left(u \frac{\partial w}{\partial r}+w \frac{\partial w}{\partial z}\right)=-\frac{\partial P}{\partial z}+\mu\left\{\frac{1}{r} \frac{\partial}{\partial r}\left(r \frac{\partial w}{\partial r}\right)+\frac{\partial^{2} w}{\partial z^{2}}\right\}+\rho g \alpha\left(T-T_{0}\right) \\
\rho c_{p}\left(u \frac{\partial T}{\partial r}+w \frac{\partial T}{\partial z}\right)=k\left(\frac{\partial^{2} T}{\partial r^{2}}+\frac{1}{r} \frac{\partial T}{\partial r}+\frac{\partial^{2} T}{\partial z^{2}}\right)+b_{1} k \frac{\partial T}{\partial r}
\end{gathered}
$$

Where $u, w$ are the velocity in the direction $r$ and $z$ respectively, $P$ is the pressure, $T$ is the temperature, $T_{0}$ Initial temperature of liquid, $Q_{0}$ is the constant heat addition/absorption, $c_{p}$ is the specific heat at constant pressure, $k$ is the thermal conductivity, $b_{1}$ is the source or sink flow and $\rho$ is the density of the fluid,. Introducing the dimensionless variables as follows:

$$
\begin{gathered}
\bar{r}=\frac{r}{a}, \bar{z}=\frac{z}{\lambda}, \bar{w}=\frac{w}{c}, \bar{u}=\frac{u}{c \delta}, \bar{p}=\frac{p a^{2}}{\mu c \lambda}, \\
\bar{\theta}=\frac{T-T_{0}}{T_{0}}, \bar{\delta}=\frac{a}{\lambda}, h=1+e \sin (2 \pi z), e=\frac{b}{a}, \\
R_{e}=\frac{\rho c a}{\mu}, G=\frac{a^{3} g \alpha T_{0}}{v^{2}}, \operatorname{Pr}=\frac{\mu c_{p}}{k} \beta=\frac{a^{2} Q_{0}}{k T_{0}}, a_{l}=\frac{k}{\rho c_{p}},
\end{gathered}
$$

where, $R_{e}$ is the Reynolds number, $\delta$ is the wave number, $e$ is the amplitude ratio, $G_{r}$ is the Grashof number, $\operatorname{Pr}$ is the Prandtl number, $a_{l}$ latent heat, and $\beta$ is the non-dimensional heat source parameter. Substituting by the equation (6) into the equations (2-5), we obtain

$$
\begin{gathered}
\frac{1}{r} \frac{\partial}{\partial r}(r u)+\frac{\partial w}{\partial z}=0 \\
R_{e} \delta^{3}\left(u \frac{\partial u}{\partial r}+w \frac{\partial u}{\partial z}\right)=-\frac{\partial P}{\partial r}+\delta^{2}\left\{\frac{1}{r} \frac{\partial}{\partial r}\left(r \frac{\partial u}{\partial r}\right)-\frac{u}{r^{2}}+\delta^{2} \frac{\partial^{2} u}{\partial z^{2}}\right\} \\
R_{e} \delta\left(u \frac{\partial w}{\partial r}+w \frac{\partial w}{\partial z}\right)=-\frac{\partial P}{\partial z}+\frac{1}{r} \frac{\partial}{\partial r}\left(r \frac{\partial w}{\partial r}\right)+\delta^{2} \frac{\partial^{2} w}{\partial z^{2}}+G \theta \\
c a \delta\left(u \frac{\partial \theta}{\partial r}+w \frac{\partial \theta}{\partial z}\right)=a_{l}\left(\frac{\partial^{2} \theta}{\partial r^{2}}+\frac{1}{r} \frac{\partial \theta}{\partial r}+\delta^{2} \frac{\partial^{2} \theta}{\partial z^{2}}\right)+a_{l} b_{1} a \frac{\partial \theta}{\partial r}
\end{gathered}
$$

When the wavelength is large $(\delta \ll 1)$, the Reynolds number is quite small $\left(R_{e} \rightarrow 0\right)$ and the equations (8-10) becomes

$$
\begin{gathered}
\frac{\partial P}{\partial r}=0 \\
\frac{\partial P}{\partial z}=\frac{1}{r} \frac{\partial}{\partial r}\left(r \frac{\partial w}{\partial r}\right)+G_{r} \theta \\
a_{l}\left(\frac{\partial^{2} \theta}{\partial r^{2}}+\frac{1}{r} \frac{\partial \theta}{\partial r}\right)+a_{l} b_{1} a \frac{\partial \theta}{\partial r}=0 .
\end{gathered}
$$

The dimensionless volume flow rate in the fixed frame of reference is given by

$$
q=2 \int_{0}^{h} w r d r
$$

$$
\begin{array}{llrl}
\frac{\partial w}{\partial r}=0 \quad \text { at } & r=0 \\
w=-1 \quad \text { at } & r=h \\
\frac{\partial \theta}{\partial r}=\epsilon \gamma & \text { at } & r=R \\
\theta=\theta_{0} \quad \text { at } & r=h .
\end{array}
$$

Solving Eq. (13) using the boundary conditions (17-18), then

$$
\begin{gathered}
\theta(r)=\epsilon \gamma R\left(\frac{r}{h}-1\right)+\frac{1}{a b_{1}}\left(a b_{1}(R-h)-a b_{1}(R-r)+\right. \\
\left.\frac{a^{2} b_{1}^{2}}{2}(R-h)^{2}-\frac{a^{2} b_{1}^{2}}{2}(R-r)^{2}+\theta_{0}\right),
\end{gathered}
$$

where,

$$
\gamma=\frac{\rho_{v}\left(L+\left(c_{l}-c_{v}\right) \Delta \theta_{0}\right)}{T_{0} k_{l}}
$$

substituting by Eq. (19) into the Eq. (12) and solving Eq. (12) with the boundary conditions (15-16), then

$$
\begin{gathered}
w(r)= \\
\frac{1}{4} \frac{d p}{d z}\left(r^{2}-h^{2}\right)-G_{r}\left(\epsilon \gamma R\left(\frac{r^{3}}{9 h}-\frac{r^{2}}{4}\right)+\frac{1}{a b_{1}}\left(\frac{a b_{1} r^{2}}{4}(R-h)-\right.\right. \\
a b_{1}\left(\frac{R r^{2}}{4}-\frac{r^{3}}{9}\right)+\frac{a^{2} b_{1}^{2} r^{2}}{4}(R-h)^{2}-\frac{a^{2} b_{1}^{2}}{2}\left(\frac{R^{2} r^{2}}{4}-\frac{2 R r^{3}}{9}-\right. \\
\left.\left.\left.\frac{r^{4}}{16}\right)\right)+\frac{\theta_{0} r^{2}}{4}-M\right)-1,
\end{gathered}
$$

where,

$$
\begin{gathered}
M=\epsilon \gamma R\left(\frac{-5 h^{2}}{36}\right)+\frac{1}{a b_{1}}\left(\frac{a b_{1} h^{2}}{4}(R-h)-a b_{1}\left(\frac{R h^{2}}{4}-\frac{h^{3}}{9}\right)+\right. \\
\left.\frac{a^{2} b_{1}^{2} h^{2}}{4}(R-h)^{2}-\frac{a^{2} b_{1}^{2}}{2}\left(\frac{R^{2} h^{2}}{4}-\frac{2 R h^{3}}{9}-\frac{h^{4}}{16}\right)+\frac{\theta_{0} h^{2}}{4}\right),
\end{gathered}
$$

The volume flow rate is given by

$$
q=-\frac{d p}{d z}\left(\frac{h^{4}}{8}\right)+\frac{G \beta h^{6}}{96}-h^{2} .
$$

On the basis of continuity Eq. (2), we find that, the velocity of cylindrical coordinates of the vapour bubble [22, 24], can be written as

$$
w(r, t)=\frac{\epsilon R \dot{R}}{r},
$$

where, $R$ is the instantaneous bubble radius, $\dot{R}$ is the instantaneous radial velocity of bubble boundary, $t$ is the time of bubble growth, $\epsilon=1-\rho_{v} / \rho_{l}$ is the density ratio, and $\rho_{v}$, $\rho_{l}$ are the density of vapour and liquid respectively.

From the Eq. (22), we can obtain the velocity of vapour bubble radius in a vertical cylindrical tube as the form

$$
\begin{gathered}
\dot{R}(r, t)=\frac{1}{\epsilon}\left(\frac{1}{4} \frac{d p}{d z}\left(R^{2}-h^{2}\right)-G_{r}\left(\epsilon \gamma R\left(\frac{R^{3}}{9 h}-\frac{R^{2}}{4}\right)+\right.\right. \\
\frac{1}{a b_{1}}\left(\frac{a b_{1} r^{2}}{4}(R-h)-a b_{1}\left(\frac{R^{3}}{4}-\frac{R^{3}}{9}\right)+\frac{a^{2} b_{1}^{2} R^{2}}{4}(R-h)^{2}+\right. \\
\left.\left.\left.\frac{5 a^{2} b_{1}^{2}}{288} R^{4}\right)+\frac{\theta_{0} R^{2}}{4}-M\right)-1\right) .
\end{gathered}
$$




$$
\begin{array}{r}
R(t)=\frac{\sqrt{-4-A h^{2}}}{\sqrt{\bar{A}}} \tan \left[\frac { \sqrt { A } \sqrt { - 4 - A h ^ { 2 } } } { \mathbf { 4 } \epsilon } \left(\frac{4 \epsilon \tan ^{-1}\left(\frac{\sqrt{A} R_{0}}{\sqrt{-4-A h^{2}}}\right)}{\sqrt{A} \sqrt{-4-A h^{2}}}+\right.\right. \\
\left.\left.\left(t-t_{0}\right)\right)\right]
\end{array}
$$

where,

$$
A=\frac{G_{r} \beta h^{2}}{6} \frac{-8 q}{h^{4}}-\frac{8}{h^{2}}
$$

\section{Discussion and Results}

The physical problem is described by mass, momentum, and heat equations (2), (3), (4) and (5) respectively in Newtonian fluid throughout the growth process. The problem is solved analytically to obtain the velocity and temperature distribution of the flow under the effect of some physical parameters such as, Grashof number $G_{r}$, heat source parameter $\beta$, density ratio $\epsilon$, and amplitude ratio $e$. The growth of vapour bubble of Newtonian fluids in a vertical cylindrical tube as shown in Figure 1 with two-phase density and it is obtained by relation (24).The physical values are calculated by Haar et al. [28] as given by Table 1.In Figure 2, the velocity distribution in terms of $r$ for two different values of density ratio $(\epsilon=0.6,0.7)$, it is clearly, the velocity distribution is proportional inversely with the increasing of density ratio $\epsilon$. The velocity distribution in terms of $r$ with two different values of heat source parameter $\beta(\beta=5,5.5)$ as shown in Figure 3. It is observed that, the velocity distribution decreasing with the increasing of heat source parameter $\beta$. On the contrary, the velocity distribution in terms of $r$ with two different values of Grasof number $G_{r}\left(G_{r}=4.5,5\right)$ as shown in Figure 4. It is observed that, the velocity distribution is proportional with increasing the Grashof number $G_{r}$. The behavior of velocity distribution is agreement with Ref. [9]. Temperature distribution in terms of $r$ is shown in Figures 5 and 6, it is clearly the temperature distribution increasing with the increasing of amplitude ratio $e(e=0.4,0.6)$ when the source flow $b=1$, and decreasing with increasing of amplitude ratio $e$ when sink flow $b=-1$. The velocity of bubble radius $\dot{R}$ is plotted as a function of $R$ as shown in Figures 7, 8. It is observed the velocity of bubble radius decreasing with the increasing of $R$ and shifted for lower values with increasing the density ratio $\epsilon(\epsilon=0.6,0.7)$ and heat source parameter $\beta(\beta=5,5.5)$ respectively. In Figure 9, the velocity of bubble radius $\dot{R}$ in terms of $R$, it is observed that $\dot{R}$ increasing with the increasing of Grashof number $G_{r}\left(G_{r}=4.5,5\right)$. The growth of vapour bubble in a superheated liquid in a vertical cylindrical tube is shown in Figures 10 and 11, the bubble radius in terms of time $t$ with two different values of amplitude ratio $e(e=0.6,0.7)$ and density ratio $\epsilon(\epsilon=0.5,0.7)$ respectively. It is clearly the bubble radius is proportional inversely with the increasing of amplitude ratio $e$ and density ratio $\epsilon$ and this result is

\begin{tabular}{|c|c|c|c|c|c|}
\hline Parameter & Value & Unite & Parameter & Value & Unite \\
\hline$\rho_{v}$ & $0.597[12]$ & $\mathrm{kg} / \mathrm{m}^{3}$ & $k$ & $0.6857[28]$ & $W / m k^{0}$ \\
\hline$\rho_{l}$ & $958.3[12]$ & $\mathrm{kg} / \mathrm{m}^{3}$ & $\sigma$ & $0.0535[28]$ & $\mathrm{kg} / \mathrm{s}^{2}$ \\
\hline$R_{0}$ & $1 \times 10^{-5}$ & $m$ & $\Delta \theta_{0}$ & 273.15 [29] & $k^{0}$ \\
\hline$c_{p}$ & 4240 [12] & $J\left(k g k^{0}\right)$ & $T_{0}$ & $100[6]$ & $k^{0}$ \\
\hline$L$ & 533000 & $J k g$ & & & \\
\hline$\dot{R_{0}}$ & $10^{-3}$ & $\mathrm{~m} / \mathrm{s}$ & & & \\
\hline$R_{m}$ & $10^{-4}$ & $m$ & & & \\
\hline
\end{tabular}
agreement with Ref. [10].

Table 1. Parameters values used in the present problem.

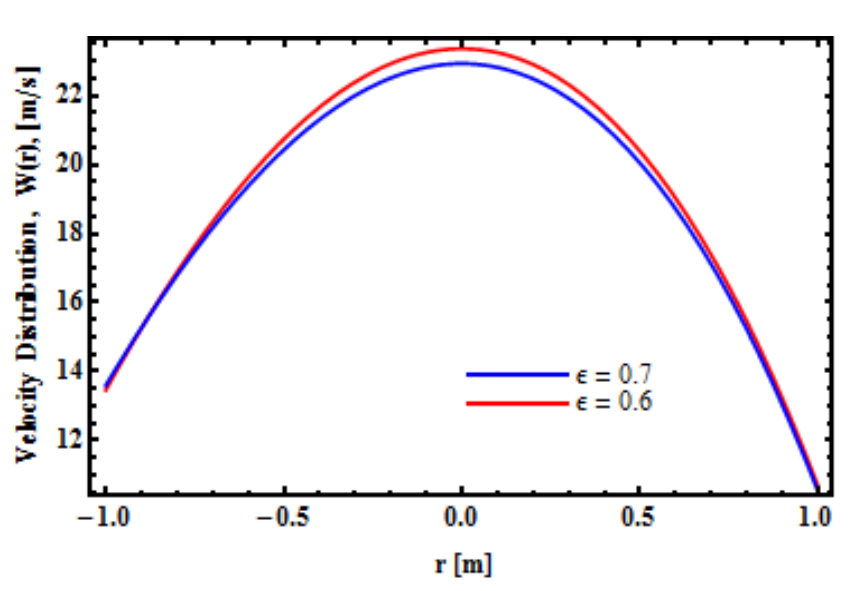

Figure 2. The Velocity distribution in terms of $r$ for the different values of density ratio $\epsilon(\epsilon=0.6,0.7)$.

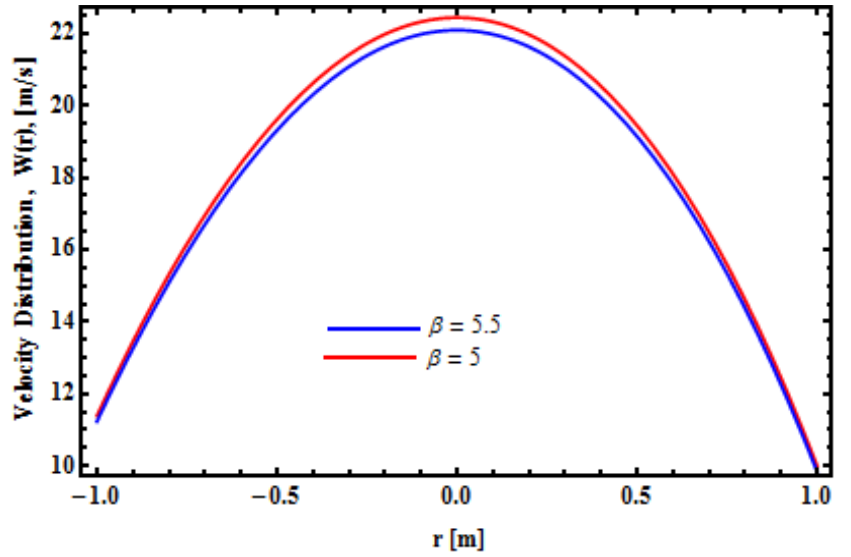

Figure 3. The Velocity distribution in terms of $r$ for the different values of heat source $\beta(\beta=5.5,5)$. 


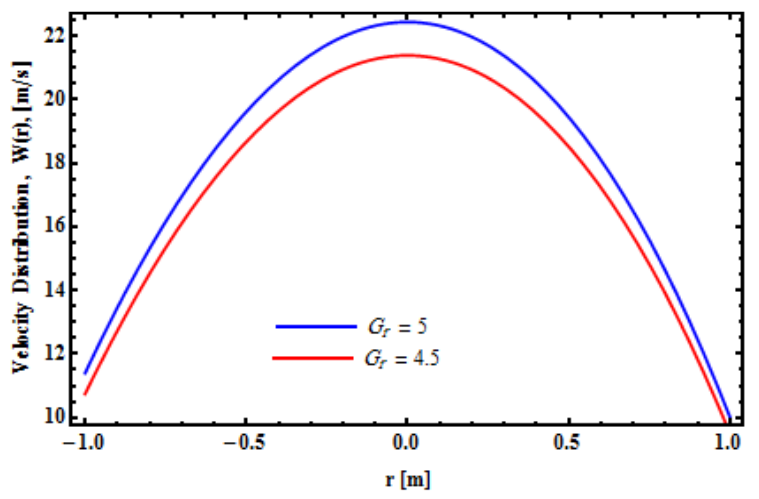

Figure 4. The Velocity distribution in terms of $r$ for the different values of Grashof number $G_{r}\left(G_{r}=5.5,5\right)$.

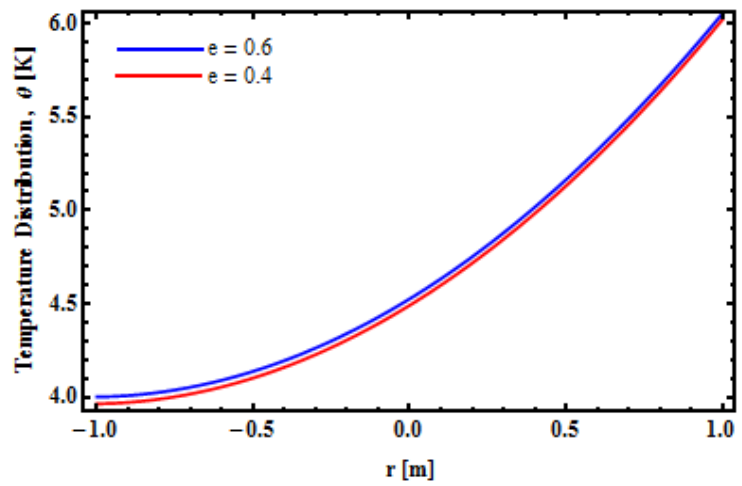

Figure 5. Temperature distribution $\theta(r, z)$ in terms ofr in case $b_{1}=1$ for different value of amplitude ratio $e(e=0.4,0.6)$.

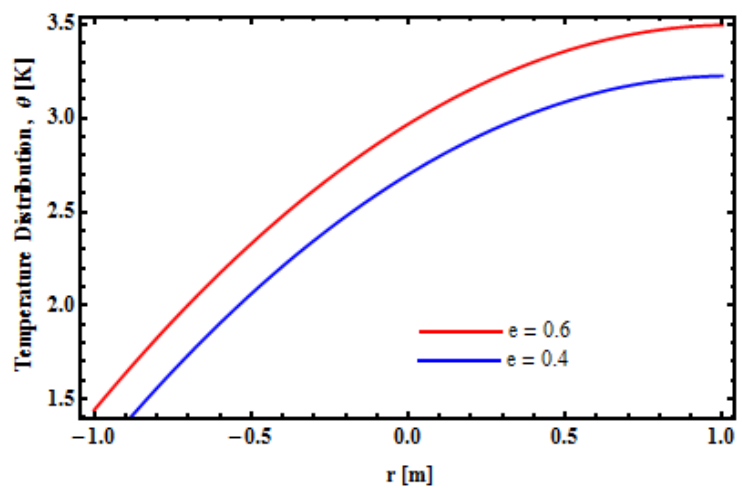

Figure 6. Temperature distribution $\theta(r, z)$ in terms of $r$ in case $b_{1}=-1$ for different values of amplitude ratio $e(e=0.4,0.6)$.

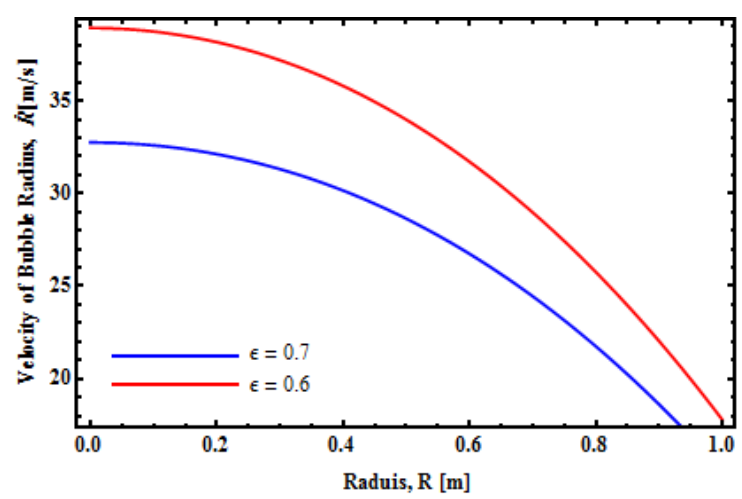

Figure 7. The velocity of bubble radius in terms of radius $R$ for different valuesof density ratio $\epsilon(\epsilon=0.7,0.6)$.

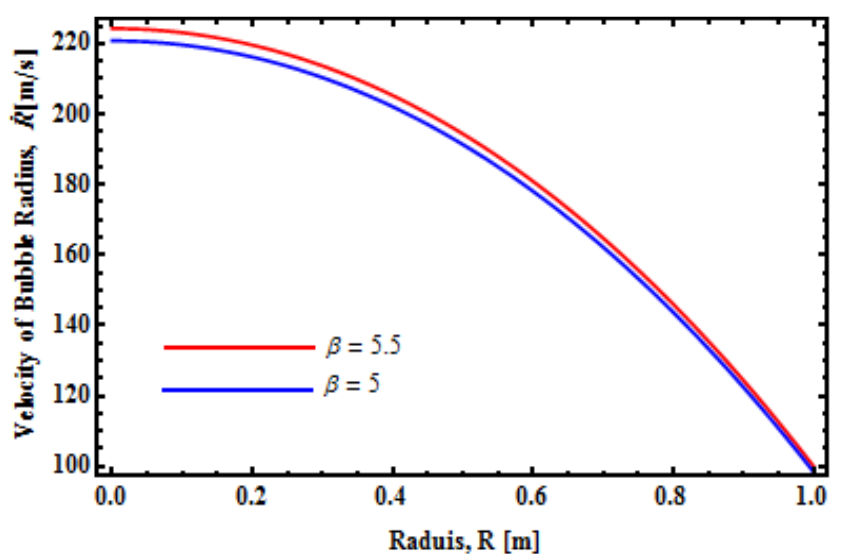

Figure 8. The velocity of bubble radius in terms of radius $R$ for different values of heat source parameter $\beta(\beta=5,5.5)$.

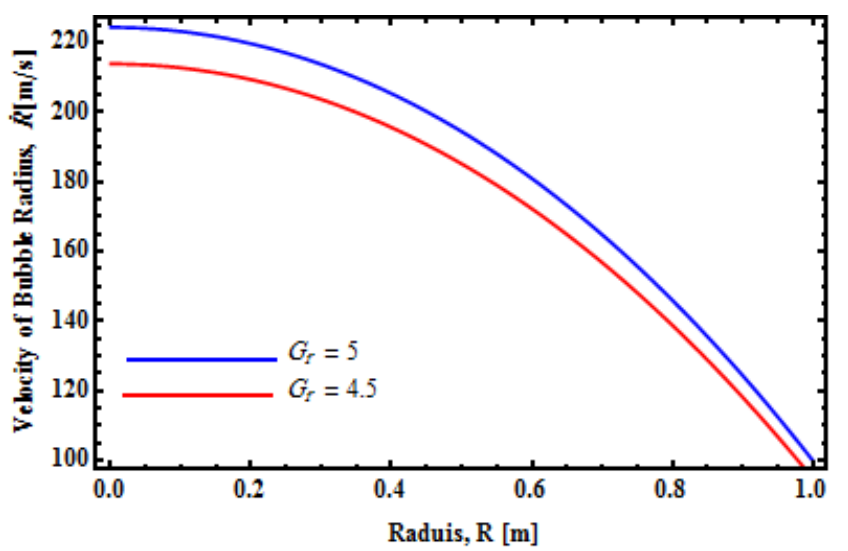

Figure 9. Thevelocity of bubble radius in terms of radius $R$ for different values of heat Grashof number $G_{r}\left(G_{r}=5,4.5\right)$.

In the Figures 12 , and 13 the growth of vapour bubbles is plotted in terms of density ratio $\epsilon$, and amplitude ratio $e$ respectively. It is clearly, the radius of vapour bubble $R(t)$ is proportional inversely with density ratio $\epsilon$, and amplitude ratio $e$, this results is agreement with Mohammadein and Gouda [27].

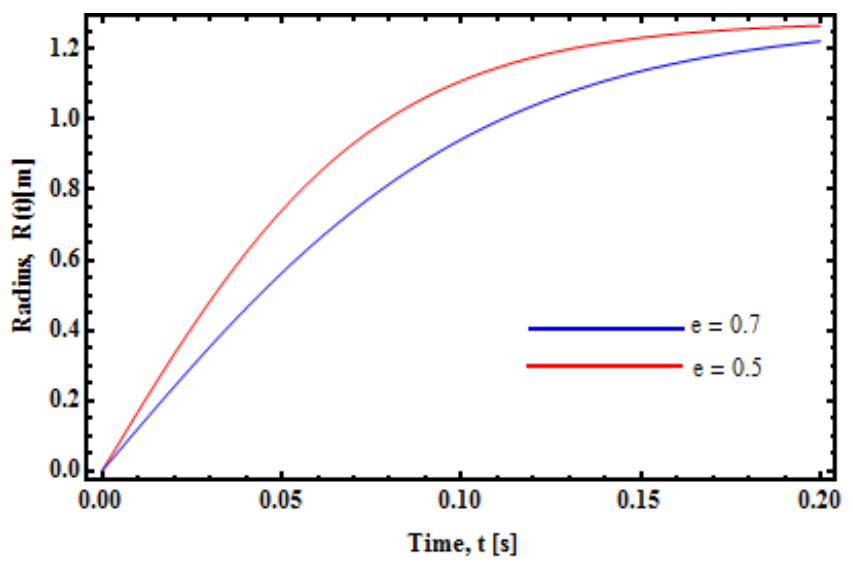

Figure 10. The radius of vapour bubble in terms of time tfor different values of amplitude ratioe $(e=0.7,0.6)$. 


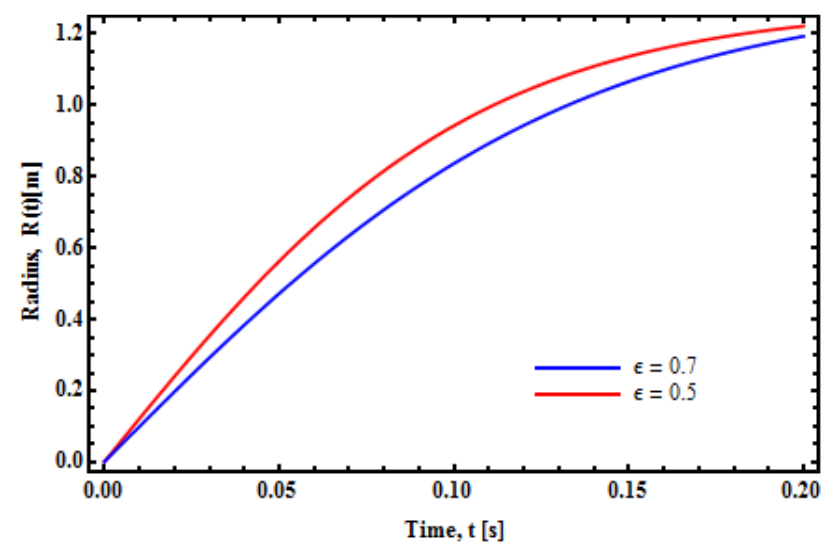

Figure 11. The radius of vapour bubble in terms of time $t$ For different values of density ratio $\epsilon=0.7,0.5)$.

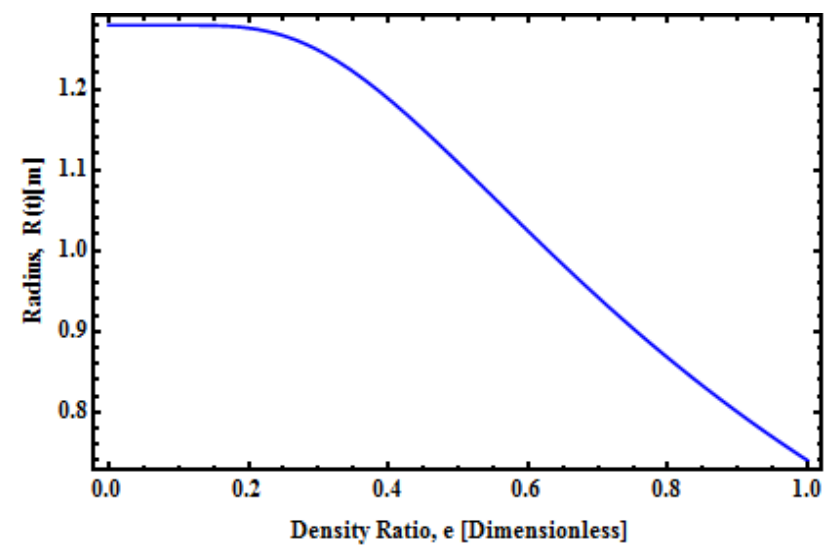

Figure 12. The relation between the bubble radius $R$ and the density ratio $\epsilon$ through its range, $0<\epsilon<1$.

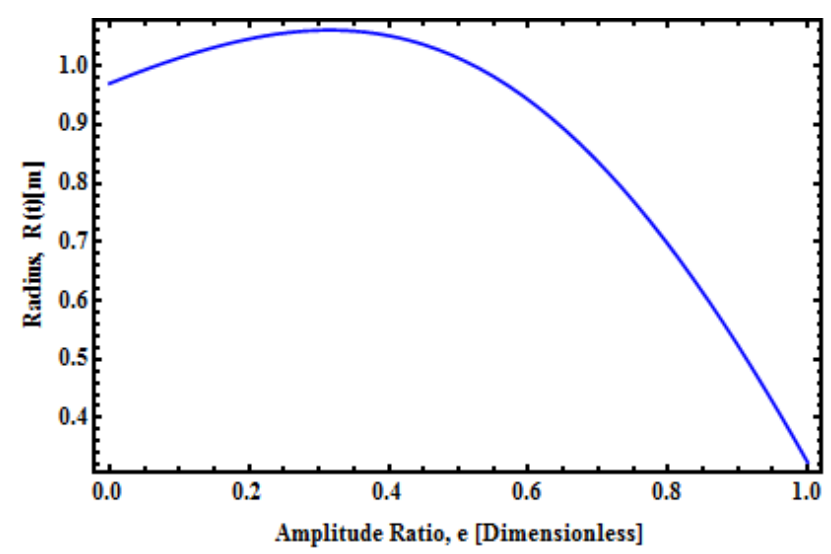

Figure 13. The relation between the bubble radius $R$ and the amplitude ratio $e$ through its range, $0<e<1$.

\section{Conclusion}

Growth of a vapour bubble is solved analytically under the effect of peristaltic motion long wavelength, low Reynolds number, and other physical parameters, taking into account the effect of density ratio $\epsilon$, amplitude ratio $e$.The growth of vapour bubble under the effect of peristaltic motion is given by Eqs. (24) with two-phase density. The values of physical parameters are given by Table (1). The discussion of results and figures concluded the following remarks: (a) The growth of vapour bubble radius is proportional inversely with amplitude ratio, and density ratio $\epsilon$.

(b) The velocity of vapour bubble is proportional with amplitude ratio $e$, densityratio $\epsilon$, and Grashof number $G_{r}$.

(c) The relation between the bubble radius $R$ with the density ratio $\epsilon$, amplitude ratio $e$ gives better agreements with Ref. [27]. The above concluded remarks prove the validity of the proposed model, and how to extend the present model in more properties of fluid and flow.

\section{Acknowledgements}

The authors are grateful to the reviewers for their useful comments.

\section{References}

[1] K. Ramesh and M. Devakar. Peristaltic Transport of MHD Walter's fluid through porous medium with heat transfer. Int. J. of Biomathematics, 11, 34 (2014).

[2] K. Das. Effects of Slip and Heat Transfer on MHD Peristaltic Flow in an Inclined Asymmetric Channel. Iran. J. of Math. Scie. and Info. 7, 35 (2012).

[3] A. V. Mernone, J. N. Mazumdar. A Mathematical Study of Peristaltic Transport of a Casson Fluid. Math. and Comp. Mode. 35, 895 (2002).

[4] T. Hayat, Y. Wang, A. M. Siddiqui, K. Hutter, S. Asghar. Peristaltic Transport of a Third-Order Fluid in a Circular Cylindrical Tube. Math. Mode. And Meth. In Appl. Scie. 12, 1691 (2002).

[5] N. Divinis, T. D. Karapantsios, M. Kostoglou, C. S. Panoutsos, V. Bontozoglou, A. C. Michels. Bubbles growing in supersaturated solutions at reduced gravity. AIChE. J. 50, $23692(004)$.

[6] N. Divinis, T. D. Karapantsios, R. De Bruijn, M. Kostoglou, V. Bontozoglou, J-C. Legros. Bubble dynamics during degassing of liquids at microgravity conditions. AIChE.J.,52, 3029, (2006).

[7] N. Divinis, M. Kostoglou, T. D. Karapantsios, V. Bontozoglou. Self-similar growth of a gas bubble induced by localized heating: the effect of temperature-dependent transport properties. Chem. Engi. Scie. 60, 1673,(2005).

[8] N. Divinis, T. Karapantsios, M. Kostoglou, V. Bontozoglou, R. de Bruijn, J. Legros. Lateral motion and interaction of gas bubbles growing over spherical and plate heaters. Microgravity Sci Tech. 18, 204 (2006).

[9] T. D.Karapantsios, M. Kostoglou, N. Divinis, V. Bontozoglou. Nucleation, growth and detachment of neighboring bubbles over miniature heaters. Chem.Engi.Sci. 63, 3438 (2008).

[10] T. D. Karapantsios, M. Kostoglou, S. P. Evgenidis. From single bubbles on solid surfaces to massive bubbly flows during decompression sickness. Proceedings of the Symposium "Life in Space for Life on Earth" (ESA, SP-663), 22 (2008)[Angers, France].

http://esamultimedia.esa.int/multimedia/publications/SP663/SP-663-toc.pdf. 
[11] S. P. Evgenidis, N. A.Kazakis, T. D. Karapantsios. Bubbly flow characteristics during decompression sickness: effect of surfactant and electrolyte on bubble size distribution. Colloids Surf A Physicochem Eng. Asp. 365, 46 (2010).

[12] M. A. Chappell, S, J. Payne. A physiological model of the interaction between tissue bubbles and the formation of bloodborne bubbles under decompression. Phys. Med. Biol. 51, 2321 (2006).

[13] H. D. Van Liew, M. E. Burkard. Density of decompression bubbles and competition for gas among bubbles, tissue, and blood. J. Appl. Physiol.75, 2293 (1993).

[14] O. M. F. R. S. Lord and Rayleigh. Philos. On the pressure developed in a liquid during the collapse of a spherical cavity. Mag. 34, 94 (1917).

[15] M. S. Plesset and S. A. Zwick. A Non steady Heat Diffusion Problem with Spherical Symmetry. J. Appl. Phys. 23, 95 (1952).

[16] M. S. Plesset and S. A. Zwick. On the dynamics of small vapor bubbles in liquid. J. Appl. Phys. 25, 493 (1954).

[17] H. K. Forster and N. Zuber. J. Appl. Phys. 25, 474 (1954).

[18] P. Dergarabedian. The Rate of Growth of Vapour Bubbles in Superheated Water. J. Appl. Mech., 20, 537 (1953).

[19] L. E. Scriven. On the Dynamics of Phase Growth. Chem. Engi. Scie. 10, 1 (1959).

[20] B. B. Mikic, W. M. Rohsenow, P. Griffith. On Bubble Growth Rates, Int. J. Heat Mass Trans., 13, 657 (1970).
[21] M. A. Lang, N. E. Smith. Proceedings of the Advanced Scientific Diving Workshop, February 23-24, 2006, Smithsonian Institution, Washington, DC, 277 (2006).

[22] S. A. Mohammadein and K. G. Mohamed. Growth of a vapour bubble in a viscous, superheated liquid in two-phase flow. Cana. J. of Phys. 93, 1 (2015).

[23] S. A. Mohammadein and Sh. A. Gouda. Temperature distribution in a mixture surrounding a growing vapour bubble. Heat Mass Transfer, 42, 359 (2006).

[24] S. A. Mohammadein and R. A. Gad El-Rab. The growth of vapour bubble in superheated water between two-finite boundaries. Cana. J. of Phys. 79, 1021 (2001).

[25] S. A. Mohammadein and A. K. Abu-Nab. Growth of Vapour Bubble Flow inside a Symmetric Vertical Cylindrical Tube. Fluid Mech. 2(2), 28 (2016).

[26] S. A. Mohammadein and A. K. Abu-Nab. The Growth of Vapour Bubble between two-Phase Peristaltic Bubbly Flow inside a Vertical Cylindrical Tube. Int. J. Thin. Fil. Sci. Tec. 6, 29 (2017).

[27] S. A. Mohammadein and K. G. Mohamed. Growth of a Vapour Bubble in a Superheated Liquid of Variable Surface Tension and Viscosity Between Two-phase Flow. Appl. Math. Inf. Sci. 7, (6), 2311 (2013).

[28] L. Haar, J. S. Callagher, Kell G. S. NBS/NRV, Steam tables, (1984). 